Hardarik Blühdorn, Tinka Reichmann

\title{
Adverbiale Satzverknüpfungen im Portugiesischen und Deutschen: Schnittstellen zwischen Syntax, Semantik und Diskurs
}

\begin{abstract}
This article deals with finite adverbial subordinate clauses and adverbial infinitivals. In Portuguese, as in other Romance languages, adverbial infinitivals are used more frequently and for a greater diversity of functions than in German. The article focuses on the semantic and textual differences between finite clauses and infinitivals. It is shown that infinitivals, by means of their introducing prepositions, link the described state of affairs to the surrounding text (sentence coherence). As they lack a verbal mood component, they cannot connect the state of affairs to the speaker and the ongoing discourse. Finite adverbial clauses are linked to their syntactic context by means of subordinating conjunctions. In addition, they are connected deictically to the speaker and the ongoing discourse by the verbal tense and mood components (discourse coherence).
\end{abstract}

Keywords: Adverbial clauses, infinitivals, sentence/discourse coherence, contrastive grammar

\footnotetext{
Prof. Dr. Hardarik Blühdorn: Institut für Deutsche Sprache, R 5, 6-13, 68161 Mannheim, E-Mail: hardarik@ids-mannheim.de

Prof. Dr. Tinka Reichmann: Universidade de São Paulo, Departamento de Letras Modernas, Av. Prof. Luciano Gualberto, 403, BR-05508-900 São Paulo, E-Mail: reichmann@usp.br
}

\section{Einleitung}

Thema dieses Aufsatzes sind adverbiale Satzverknüpfungen, insbesondere Konjunktionen und Präpositionen als lexikalische Satzverknüpfer sowie Tempus und Modus als grammatische Diskursverknüpfungsmittel. Ferner wird mit der Fokussierung bzw. Fokussierbarkeit von Subjekten ein Aspekt der Informationsstruktur berührt. Im Mittelpunkt der Untersuchung steht das Portugiesische, das im Bereich der Satzverknüpfungen verglichen mit anderen (romanischen und nicht-romanischen) Sprachen über eine besonders große Ausdrucksvielfalt verfügt (cf. Rudolph 
1997; Gärtner 2003; Blühdorn 2012b). Zum Vergleich ziehen wir das Deutsche heran, dessen Satzverknüpfungsmittel deutlich weniger differenziert sind.

Adverbialia sind semantisch modifizierende Ausdrücke, die sich mit anderen (vor allem verbalen) Ausdrücken verbinden, ohne deren syntaktische Eigenschaften zu verändern und insbesondere ohne syntaktische Valenzen zu sättigen (cf. Corver/Delfitto 2000; Delfitto 2000; Bierwisch 2003; Rocha/Lopes 2009). Sie können durch Adverbien realisiert werden, aber auch durch komplexere Ausdrücke wie Präpositionalphrasen, oder durch Ausdrücke, die ein eigenes Verb enthalten, etwa Infinitivgruppen oder vollständige finite Nebensätze. In diesem Aufsatz geht es vor allem um Adverbialia der beiden letztgenannten Typen, die Verbgruppen oder Sätze erweitern.

Adverbiale Infinitivgruppen werden typischerweise durch eine Präposition eingeleitet, finite adverbiale Nebensätze durch einen Subjunktor. Zwei Beispiele für Kausalverknüpfungen:

(1) Esse doutor tinha uma grande reputação nos subúrbios, por ser chefe de seção da Secretaria dos Telégrafos. (Lima Barreto, Triste fim de Policarpo Quaresma) Dieser Doktor hatte einen ausgezeichneten Ruf in den Vorstädten, wörtl.: für sein [Verb] Referatsleiter im Telegrafenamt. ${ }^{1}$

(2) Era um homem como todos os outros, a não ser aqueles que têm ambições políticas ou de fortuna, porque Quaresma não as tinha no mínimo grau. (ib.)

Er war ein Mann wie alle anderen, abgesehen von denen, die politischen oder wirtschaftlichen Ehrgeiz haben, weil Quaresma den absolut nicht hatte.

Während adverbiale Infinitivgruppen und finite adverbiale Nebensätze im Portugiesischen als gleichberechtigte Ausdrucksmittel nebeneinanderstehen, ist der Prototyp eines verbhaltigen Adverbiales im Deutschen der finite Nebensatz:

(3) Als Gregor Samsa eines Morgens aus unruhigen Träumen erwachte, fand er sich in seinem Bett zu einem ungeheuren Ungeziefer verwandelt. (Franz Kafka, Die Verwandlung)

Adverbiale Infinitivgruppen kommen nur mit den Präpositionen um, ohne, (an)statt und außer vor und sind auf einige wenige semantische Funktionen beschränkt (cf. Duden 2009, 852). Zwei Beispiele:

1 Die angeführten Belege stammen aus literarischen Texten, aus Internetquellen und aus der berücksichtigten Fachliteratur. Die deutschen Übersetzungen der portugiesischen Belege stammen von den Autoren des vorliegenden Aufsatzes. Sie sollen geeignet sein, die jeweils besprochenen grammatischen Punkte zu verdeutlichen. Beispiele und Übersetzungen dienen insgesamt der Illustration. Eine quantitative Korpusstudie ist nicht intendiert. 
(4) Um für die sich nähernden entscheidenden Besprechungen eine möglichst klare Stimme zu bekommen, hustete er ein wenig. (ib.)

(5) Die drei Schreibenden nickten bloß, ohne aufzuschauen. (ib.)

Finite Nebensätze wie (2) und (3) sind grammatisch dadurch gekennzeichnet, dass sie stets ein eigenes Subjekt enthalten, auch wenn dieses im Portugiesischen implizit bleiben kann wie in (6):

(6) Quando entrou em casa, naquele dia, foi a irmã quem lhe abriu a porta. (Lima Barreto, Triste fim de Policarpo Quaresma)

Als er an jenem Tag nach Hause kam, war es die Schwester, die ihm die Tür öffnete.

Infinitivgruppen treten dagegen typischerweise subjektlos auf wie in (1), (4) und (5). Ihr semantisches Subjekt muss dann aus dem Kontext rekonstruiert werden. Im Portugiesischen, anders als im Deutschen, können Infinitivgruppen allerdings auch explizite Subjekte enthalten:

(7) Além do inchaço, você pode sofrer intensa falta de ar, antes de chegar o momento de uma nova diálise. (www.gamen.com.br)

Außer der Schwellung können Sie starke Atemnot verspüren, wörtl.: vor kommen der Zeitpunkt einer neuen Dialyse.

(8) Seu corpo foi transladado para São Paulo e o trem especial no qual viajou parou a cada estação a fim de o ilustre político receber as últimas homenagens. (www.pedrodetoledo.sp.gov.br)

Sein Leichnam wurde nach São Paulo überführt, und der Sonderzug, in dem er reiste, hielt an jedem Bahnhof, wörtl.: zwecks der berühmte Politiker empfangen die letzten Ehrungen.

Darüber hinaus besitzt das Portugiesische das oft erwähnte Exotikum des flektierten Infinitivs (cf. Cunha/Cintra 2008, 426). Das sind Formen, die zwar keine Tempus- und Modusmorpheme aufweisen, wohl aber Personal- und Numerusmorpheme:

(9) Seis crianças foram hospitalizadas em Lille, no norte de França, depois de terem comido hambúrgueres congelados. (www.jn.pt)

In Lille, Nordfrankreich, wurden sechs Kinder ins Krankenhaus eingeliefert, wörtl.: nach haben gegessen tiefgefrorene Hamburger.

Eine naheliegende Frage, über die in der Literatur wiederholt gerätselt wurde, lautet: Sind finite adverbiale Nebensätze und adverbiale Infinitivgruppen im Portugiesischen reine Ausdrucksvarianten, die die gleichen semantischen und textu- 
ellen Funktionen erfüllen, oder bestehen zwischen ihnen funktionale Unterschiede, und wenn ja, welche (cf. Blühdorn/Reichmann 2010, 35s.)?

\section{Drei Hypothesen}

\subsection{Ausdrucksvarianten ohne Funktionsunterschied}

Traditionelle Grammatiken wie Vilela/Koch (2001, 378ss.) oder Cunha/Cintra (2008, 623ss.) tendieren dazu, finite adverbiale Nebensätze und adverbiale Infinitivgruppen (sog. orações subordinadas adverbiais reduzidas de infinitivo) als Ausdrucksvarianten ohne Funktionsunterschied nebeneinanderzustellen. Cunha/ Cintra $(2008,503 s$.) weisen immerhin auf funktionale Unterschiede zwischen flektiertem und nicht-flektiertem Infinitiv hin. Sie präsentieren den flektierten Infinitiv vorrangig als Mittel zur Hervorhebung des Agens und siedeln seinen Gebrauch auf stilistischer Ebene an:

(10) Aqueles homens gotejantes de suor, bêbedos de calor, desvairados de insolação, a quebrarem, a espicaçarem, a torturarem a pedra, pareciam um punhado de demônios revoltados na sua impotência contra o impassível gigante. (Aluísio Azevedo, O cortiço; aus: Cunha/Cintra 2008, 503)

Jene Männer, tropfend vor Schweiß, trunken vor Hitze, übergeschnappt vom Sonnenstich, wie sie den Stein zerbrachen, durchbohrten, folterten, wirkten wie eine Handvoll Dämonen, die sich in ihrer Ohnmacht gegen den unverwundbaren Riesen auflehnten.

In Beispielen wie diesem wird durch die flektierten Infinitive die Identität der Subjekte von Hauptsatz und Infinitiven deutlich gemacht. Gleichzeitig wird die Darstellung dadurch lebendiger, dass die wiederholten flektierten Formen den Interpreten jedes Mal anregen, sich die hart arbeitenden Männer konkret vorzustellen. Offensichtlich ist ein solches stilistisches Potenzial des flektierten Infinitivs, das Cunha/Cintra mit Begriffen wie «Hervorhebung» und «Harmonie» beschreiben, aber nur schwer operationalisierbar.

\subsection{Bekannte vs. neue Information}

Auch Marques (2010, 153ss.) nimmt an, dass sich finite Nebensätze semantisch prinzipiell nicht von Infinitivgruppen unterscheiden. Allerdings seien Infinitive nicht dafür geeignet, neue Information in den Diskurs einzuführen, sondern könnten nur verwendet werden, um schon bekannte Information mit dem Hauptsatz zu verknüpfen. Sein Beispielpaar (ib.) lautet: 
(11) A Ana ficou em casa porque estava doente.

Anna blieb zu Hause, weil sie krank war.

(12) A Ana ficou em casa por estar doente.

Anna blieb zu Hause wörtl.: für sein krank.

Beide Varianten sind nach Marques für Mitteilungskontexte geeignet, in denen schon bekannt ist, dass Anna krank war. In Kontexten, in denen diese Information nicht gegeben ist, sei nur die erste Variante verwendbar.

Diese These lässt sich allerdings ohne Schwierigkeiten widerlegen, etwa durch Beispiel (1), in dem die Information, dass der besprochene Doutor Bulhões Referatsleiter im Telegrafenamt ist, dem Leser mit der Infinitivgruppe erstmals mitgeteilt wird. Bei dem folgenden Beispiel handelt es sich um Überschrift und ersten Satz einer Pressemeldung. Hier wird gleich zweimal mit Infinitivgruppen neue Information eingeführt:

(13) Trabalhadora despedida por estar doente deve ser indenizada

A Companhia Industrial de Desenvolvimento Urbano de Cruz Alta (Cidusa) foi condenada a indenizar por danos morais uma auxiliar de limpeza despedida por ser portadora do vírus HIV. (www.trt4.jus.br)

wörtl.: Für sein krank entlassene Arbeiterin bekommt Entschädigung

Die Companhia Industrial de Desenvolvimento Urbano de Cruz Alta (Cidusa) wurde verurteilt, einer Putzfrau, die entlassen worden war, wörtl.: für sein Trägerin des HIVVirus, Schmerzensgeld zu zahlen.

\subsection{Sachverhalts- vs. Propositions- vs. Sprechaktverknüpfungen}

Peres/Mascarenhas (2006, 162s.) haben - ebenfalls am Beispiel von Kausalverknüpfungen - die These entwickelt, Infinitivgruppen mit por seien bevorzugt als Beschreibungen von Sachverhaltsursachen im Sinne von Sweetser (1990, 76ss.) zu lesen:

(14) A cidade ficou deserta por ser feriado e por estar a chover.

Die Stadt war wie leergefegt wörtl.: für sein Feiertag und für sein am Regnen.

In der Tat können die Kausalangaben in (14) nur als Angaben von Ursachen für das Eintreten des im Hauptsatz beschriebenen Sachverhalts gedeutet werden. Neves (2000, 804s.) weist darauf hin, dass finite Nebensätze mit porque dagegen 
in der Regel auch epistemische und/oder deontische Interpretationen erlauben oder sogar nahelegen:

(15) Não deve ter havido nada porque seria a primeira pessoa a tomar conhecimento disto.

Es wird schon nichts gewesen sein, da ich der erste wäre, den man benachrichtigt hätte.

Der Kausalsatz in diesem Beispiel muss als Argument verstanden werden, das angeführt wird, um die Aussage des Hauptsatzes plausibler zu machen (epistemische Verknüpfung). Die Verallgemeinerung könnte somit lauten, dass adverbiale Infinitivgruppen Interpretationen auf der Sachverhaltsebene im Sinne Sweetsers (1990, 76ss.) begünstigen, während finite Nebensätze Deutungen auf unterschiedlichen semantischen Ebenen erlauben.

In Blühdorn/Reichmann (2010, 35s.) wurde die These einer solchen funktionalen Arbeitsteilung als plausibel eingestuft. Zugleich wurde darauf hingewiesen, dass eine genauere Untersuchung folgen muss. Schwierigkeiten bereiten Beispiele wie (16) bis (18):

(16) Para ser sincero, tive bastante dificuldade de ler a entrevista até o final.

(www.napontadoslapis.com.br)

Um ehrlich zu sein, hatte ich ziemliche Schwierigkeiten, das Interview bis zum Ende zu lesen.

(17) Sem querer ofender, primeiro você deve aprender português!

(cascavel-parana.olx.com.br)

Ohne dich beleidigen zu wollen, lern erst mal Portugiesisch!

(18) Por falar nisso, você sabe quantos morreram hoje no Iraque?

(www.sk.com.br)

wörtl.: Für sprechen darüber (wo wir gerade beim Thema sind), wissen Sie, wie viele Tote es heute im Irak gegeben hat?

Adverbiale Infinitivgruppen wie diese können nicht als Sachverhaltsverknüpfungen, sondern ausschließlich als Sprechaktverknüpfungen im Sinne Sweetsers $(1990,76 s s$.$) gelesen werden. Sie kontextualisieren und rechtfertigen die nach-$ folgende sprachliche Handlung. 


\section{Zwei formale Oppositionen}

Im Folgenden werden die Unterschiede zwischen finiten Nebensätzen und Infinitivgruppen hinsichtlich ihrer Semantik und ihrer Diskursfunktionen genauer untersucht. Dazu wird zunächst die Formseite der Ausdrücke betrachtet.

Das Inventar der Infinitive umfasst im Deutschen wie im Portugiesischen den Infinitiv Präsens (z.B. arbeiten/trabalhar) und den Infinitiv Perfekt (gearbeitet haben/ter trabalhado). Es gibt keinen Infinitiv Präteritum. Ein Infinitiv Futur (arbeiten werden) kann im Deutschen rein formal gebildet, aber in keiner Infinitivkonstruktion verwendet werden $\left({ }^{\star}\right.$ Otto kann arbeiten werden, ${ }^{\star}$ Arbeiten werden macht glücklich, ${ }^{\star}$ Otto ist zufrieden, ohne arbeiten zu werden). Im Portugiesischen hat der periphrastische Infinitiv mit dem Futur-Auxiliar ir (ir trabalhar) keine Zukunftsbedeutung. Eine Modusunterscheidung findet beim Infinitiv in beiden Sprachen nicht statt, d.h. es gibt keinen Infinitiv Konjunktiv oder Imperativ.

Im Deutschen können Infinitive grundsätzlich kein explizites syntaktisches Subjekt haben. Demnach bestehen zwei formale Oppositionen, die fest miteinander gekoppelt sind:

(i) Finite Nebensätze sind immer für Tempus und Modus spezifiziert, Infinitivgruppen nur reduziert für Tempus und nicht für Modus.

(ii) Finite Nebensätze haben immer ein eigenes Subjekt, Infinitivgruppen nie.

Beide Oppositionen zeigen einen positiven Wert für finite Nebensätze und einen negativen für Infinitivgruppen.

Im Portugiesischen, wo Infinitive explizite syntaktische Subjekte haben können, sind die Oppositionen dissoziiert:

(i) Finite Nebensätze sind immer für Tempus und Modus spezifiziert, Infinitivgruppen nur reduziert für Tempus und nicht für Modus.

(ii) Finite Nebensätze haben immer ein eigenes Subjekt. Infinitivgruppen können ein eigenes Subjekt haben oder subjektlos sein.

Für das Deutsche ergibt sich eine zweifache, für das Portugiesische eine dreifache Unterscheidung: 
Abb. 1: Ausdrucksinventare für verbhaltige Adverbialia Deutsch-Portugiesisch

\begin{tabular}{l|l|l}
\hline Ausdrucksklasse Deutsch & formales Merkmal & Ausdrucksklasse Portugiesisch \\
\hline finite Nebensätze & $\begin{array}{l}\text { + eigenes Subjekt } \\
\text { + Tempus/Modus }\end{array}$ & finite Nebensätze \\
\cline { 2 - 3 } & $\begin{array}{l}\text { + eigenes Subjekt } \\
\text { - Tempus/Modus }\end{array}$ & subjekthaltige Infinitivgruppen \\
\cline { 2 - 3 } & $\begin{array}{l}\text { - eigenes Subjekt } \\
\text { Infinitivgruppen }\end{array}$ & subjektlose Infinitivgruppen \\
\hline
\end{tabular}

\section{Funktionale Oppositionen}

Die Oppositionen $\{ \pm$ eigenes Subjekt $\}$ und $\{ \pm$ Tempus/Modus $\}$ haben unterschiedliche semantische und textuelle Auswirkungen. In einer Sprache wie dem Deutschen, in der ihre Werte fest aneinander gekoppelt sind, kann das leicht unbemerkt bleiben. In Sprachen wie dem Portugiesischen, in denen ihre Werte voneinander unabhängiger sind, können sie besser getrennt untersucht werden.

\subsection{Subjekt}

Wenn ein verbaler Ausdruck kein eigenes formales Subjekt hat, muss die seinem Subjekt entsprechende semantische Leerstelle durch einen Referenten gesättigt werden, der aus dem Kontext erschlossen wird. Oft kommt dafür der Referent des Hauptsatzsubjekts in Frage, etwa in den oben angeführten Beispielen (12) und (16). Manchmal muss aber auch ein anderer Referent gewählt werden, etwa in (19) und (20):

(19) Die Frau stand unter Schock. Die Retter riefen erst einmal einen Arzt, um sie zu versorgen. (leicht bearbeitet aus: www.merkur-online.de)

(20) A Torre Eiffel foi construída para celebrar os cem anos da Revolução Francesa. (noticias.terra.com.br)

Der Eiffelturm wurde gebaut, um den hundertsten Jahrestag der Französischen Revolution zu feiern.

In (19) ist der Referent des Hauptsatzobjekts (einen Arzt) als Subjekt des Infinitivs einzusetzen. In (20) ist ein Referent einzusetzen, auf den im Hauptsatz nicht Bezug genommen wird (diejenigen, die den Bau initiierten). Generell verlangen 
subjektlose Ausdrücke vom Interpreten größere Inferenzleistungen als subjekthaltige Ausdrücke.

Daneben haben Subjekthaltigkeit und Subjektlosigkeit auch informationsstrukturelle Auswirkungen. Nur wo ein explizites formales Subjekt vorhanden ist, kann es durch Akzent hervorgehoben und damit im Kontext kontrastiert werden:

(21) se ELA não assinar a representação, o processo vai $\mathrm{ACABAR}^{2}$

(revistacomuniq.com.br)

wörtl.: wenn SIE nicht unterschreiben die stellungnahme, wird der prozess zum erLIEGEN kommen

In einem Satz wie (21) kann durch die Akzentuierung des Infinitivsubjekts ela ein Kontrast zu anderen möglichen Referenten hergestellt werden. Im vorliegenden Fall deutet ein solcher Kontrast auf die Möglichkeit hin, dass der erwähnte Prozess nicht zum Erliegen kommen muss, wenn eine andere Person die Unterschrift nicht leistet. In Ausdrücken, die kein explizites Subjekt aufweisen, ist eine solche Kontrastierung offensichtlich unmöglich.

Cunha/Cintra $(2008,503)$ haben die Hervorhebungsfunktion expliziter Infinitivsubjekte nur unter stilistischem Blickwinkel betrachtet. Beispiele wie (21) zeigen, dass sie sich durchaus diskurssemantisch präzisieren und damit operationalisieren lässt. Es ist zu vermuten, dass Ausdrücke ohne formales Subjekt genau dann verwendet werden, wenn der betreffende Referent aus dem Kontext erschließbar und seine Kontrastierung entbehrlich ist. Subjekthaltige Ausdrücke werden verwendet, wenn der betreffende Referent im Kontext nicht ohne Weiteres erschlossen werden kann, und insbesondere dann, wenn seine Kontrastierung gegenüber anderen möglichen Referenten erwünscht ist.

\subsection{Tempus und Modus}

Tempus und Modus haben die Funktion, das Gesagte an den Sprecher und damit an den Diskurskontext rückzubinden.

Das Tempus leistet eine solche Rückbindung in der Dimension der Zeit. Präsens-Tempora zeigen eine Überlappung des beschriebenen Ereignisses mit dem Sprechereignis, Präterital- und Futur-Tempora zeigen Entfernung vom

2 Um die Intonation wiederzugeben, werden akzentuierte Wörter in Großbuchstaben und alle übrigen Teile des Satzes in Kleinbuchstaben gesetzt. 
Sprechereignis in unterschiedlichen Richtungen auf dem Zeitstrahl an (cf. Frawley 1992, 336ss.; Klein 1994).

Der Modus leistet eine entsprechende Rückbindung in den Dimensionen der Episteme und der Deontik (cf. Lyons 1977, 787ss.; Frawley 1992, 384ss.; Diewald 1999, 172ss., 239ss.). Der Indikativ zeigt an, dass das Mitgeteilte im Einklang mit dem aktuellen Wissen des Sprechers steht, dass der Sprecher will, dass der Adressat es seinem Wissen hinzufügt, und dass der Sprecher die Verantwortung für seine Wahrheit übernimmt. Der Konjunktiv bzw. Subjunktiv - soweit er nicht vollständig grammatikalisiert und z.B. durch Rektion gefordert ist - zeigt in seinen verschiedenen Funktionsvarianten an, dass das Mitgeteilte nur den Status einer Hypothese hat, dass es sich um provisorische Information handelt, die der Adressat noch nicht ohne Weiteres seinem Wissen hinzufügen soll und/oder für deren Wahrheit der Sprecher nicht die Verantwortung übernimmt (cf. z. B. Squartini 2010). Der Imperativ zeigt an, dass der Sprecher will, dass der Adressat den beschriebenen Sachverhalt faktisch macht (cf. Blühdorn 2012a, 151ss.).

Finite Nebensätze sind im Deutschen wie im Portugiesischen obligatorisch für Tempus und Modus markiert, binden das Gesagte also stets zeitlich, epistemisch und deontisch-illokutionär an den Sprecher zurück. Infinitivgruppen sind in beiden Sprachen nicht für Modus markiert, sodass eine epistemische und deontisch-illokutionäre Rückbindung an den Sprecher durch sie nicht erfolgen kann. Soweit eine Tempusmarkierung stattfindet (beim Infinitiv Perfekt), wird durch sie meist nur Vorzeitigkeit bzw. Abgeschlossenheit des beschriebenen Sachverhalts gegenüber einem anderen im Text beschriebenen Bezugssachverhalt angezeigt, nicht aber Vorzeitigkeit oder Abgeschlossenheit gegenüber dem Sprechereignis. Somit erfolgt dann auch keine zeitliche Rückbindung an den Sprecher.

Es ist demnach zu vermuten, dass Infinitivgruppen genau dann verwendet werden, wenn eine zeitliche, epistemische und deontisch-illokutionäre Rückbindung an den Sprecher entbehrlich ist, während finite Nebensätze dann verwendet werden, wenn eine solche Rückbindung hergestellt werden soll.

\section{Diskurskohärenz und Satzkohärenz}

Sowohl adverbiale Nebensätze als auch adverbiale Infinitivgruppen leisten Beiträge zur Textkohärenz. Eine der grundlegenden Kohärenzfunktionen ist die Bereitstellung von Auswertungskontexten für Sachverhalte, Propositionen und Sprechakte (cf. Lohnstein 2004, 143; Blühdorn 2012a, 230ss.). Hierbei können zwei Verfahren unterschieden werden.

Zum einen kann innerhalb eines Satzes durch die Tempus- und Moduskategorie das Sprechereignis bzw. das aktuelle Wissens- und Normensystem des Spre- 
chers als Auswertungskontext für den beschriebenen Sachverhalt, die ausgesagte Proposition und/oder den ausgeführten Sprechakt aufgerufen werden. Zum anderen kann durch einen Subjunktor, eine Präposition oder einen anderen Konnektor ein Bezugssachverhalt, eine Bezugsproposition oder ein Bezugssprechakt als Auswertungskontext im Text eingeführt werden.

Für beide Verfahren sind zwei Arten von Kontexten zu unterscheiden:

(i) Faktizitätskontexte

(ii) Wahrheits- und Erwünschtheitskontexte

Faktizitätskontexte betreffen das Eintreten oder Nicht-Eintreten von Sachverhalten in zeitlichen Relationen zu anderen Sachverhalten. Sie werden durch Tempora und durch temporale Konnektoren bereitgestellt. Wahrheitskontexte betreffen die Richtigkeit oder Falschheit von Propositionen in Relation zu Wissenssystemen; Erwünschtheitskontexte betreffen die Legitimität oder Illegitimität von sprachlichen Handlungen in Relation zu ethischen Normen. Beide werden durch die Modi des Verbs sowie durch epistemische und deontische Konnektoren bereitgestellt.

\subsection{Bereitstellung von Auswertungskontexten durch Tempus und Modus}

Im finiten Nebensatz in (22) zeigt das Präteritum an, dass der zeitliche Kontext, für den dem Sachverhalt 'der Sprecher hat Schuhe' Faktizität abgesprochen wird, relativ zum Sprechereignis in der Vergangenheit liegt:

(22) Eu me queixava porque não tinha calçados. (www.quemdisse.com.br) Ich beklagte mich, weil ich keine Schuhe hatte.

Stünde das finite Verb des Nebensatzes im Präsens, so wäre die Deutung naheliegend, dass dem Sachverhalt Faktizität für einen zeitlichen Kontext abgesprochen wird, der zur Zeit des Sprechereignisses andauert:

(23) Eu me queixava porque não tenho calçados.

Ich beklagte mich, weil ich keine Schuhe habe.

In Beispiel (24) zeigt der Subjunktiv im Kausalsatz an, dass Faktizität des Sachverhalts 'Paula hat Angst' für einen zeitlichen Kontext, der gegenüber dem 
Sprechereignis in der Vergangenheit liegt, nur als Hypothese geprüft, nicht aber mit Wahrheitsanspruch mitgeteilt wird:

(24) Paula foi atendida num hospital universitário quando contava 11 anos, para tratar-se de problema hematológico. Em 2001, foi indicada uma cirurgia como solução para o seu caso. A família concordou, mas Paula se recusava. Não porque tivesse medo da cirurgia, mas porque dizia que queria morrer. (Francisco Paes Barreto, A angústia na psicose).

Paula musste, als sie 11 war, wegen einer Bluterkrankung in einer Universitätsklinik behandelt werden. Im Jahre 2001 wurde eine Operation verordnet, um sie zu heilen. Die Familie stimmte zu, aber Paula weigerte sich. Nicht etwa weil sie Angst vor der Operation gehabt hätte, sondern weil sie sterben wollte.

Stünde das finite Verb im Indikativ, so läge die Deutung näher, dass der Sprecher die Faktizität des Sachverhalts mit Wahrheitsanspruch mitteilt:

(25) Paula se recusou porque teve medo da cirurgia.

Paula weigerte sich, weil sie Angst vor der Operation hatte.

Faktizitäts-, Wahrheits- und Erwünschtheitskontexte, die durch Tempus- und Modusformen bereitgestellt werden, betreffen die Kohärenz zwischen dem Gesagten und dem Ereignis des Sagens. Hierfür verwenden wir den Terminus Diskurskohärenz. Zu ihr können nur solche verbalen Ausdrücke beitragen, die für Tempus und Modus markiert sind, also finite Nebensätze, nicht aber Infinitivgruppen.

\subsection{Bereitstellung von Auswertungskontexten durch Konnektoren}

Im oben angeführten Beispiel (3) fungiert der Sachverhalt, der im Nebensatz beschrieben wird ('Gregor Samsa erwacht aus unruhigen Träumen'), als Faktizitätskontext für den Sachverhalt, der im Hauptsatz beschrieben wird ('Gregor Samsa findet sich in seinem Bett zu einem ungeheuren Ungeziefer verwandelt'). Der Subjunktor als zeigt an, dass der im Hauptsatz beschriebene Sachverhalt in einem Zeitintervall der Fall ist, das sich mit dem Zeitintervall überschneidet, in dem der im Nebensatz beschriebene Sachverhalt der Fall ist. Subjunktoren wie bevor oder nachdem würden auf eine Abfolge der betreffenden Zeitintervalle hinweisen.

Die gleiche Funktion übernehmen bei Infinitivgruppen die verknüpfenden Präpositionen, etwa por in Beispiel (1), um in (4), ohne in (5), antes de in (7), a fim 
de in (8), depois de in (9) etc. Konnektoren zeigen an, dass Ausdrücke im Text Kontexte für andere Ausdrücke im Text bereitstellen. Diese Art von Kohärenz bezeichnen wir als Satzkohärenz. Zu ihr können nicht nur finite Nebensätze, sondern gleichermaßen Infinitivgruppen beitragen.

Durch Temporalkonnektoren wird Satzkohärenz auf der Sachverhaltsebene (content level im Sinne von Sweetser 1990, 76ss.) hergestellt. Konnektoren können, wie Sweetser (ib.) gezeigt hat, aber auch als Anzeiger für epistemische Relationen zwischen Propositionen oder für deontische Relationen zwischen Sprechakten verwendet und interpretiert werden. Solche epistemischen bzw. deontisch-illokutionären Konnektoren stellen Wahrheitskontexte für die gemachte Aussage bzw. Erwünschtheitskontexte für den ausgeführten Sprechakt bereit. Sie stiften Satzkohärenz auf der epistemischen und der Sprechakt-Ebene im Sinne Sweetsers (cf. Blühdorn 2008).

So rechtfertigt etwa in (26) der Konditionalsatz den Sprechakt der Meinungsäußerung:

(26) Se quiser saber minha opinião, sou contra.

(www.gazetadopovo.com.br)

Wenn Sie meine Meinung wissen wollen, ich bin dagegen.

Auch Infinitivgruppen können diese Funktion erfüllen, beispielsweise para ser sincero in (16), sem querer ofender in (17) und por falar nisso in (18).

\subsection{Vergleich}

Die folgende Tabelle gibt einen Überblick über Kohärenztypen, Kontexttypen und sprachliche Mittel für die Kontextbereitstellung:

Abb. 2: Sprachmittel und Kohärenzfunktionen

\begin{tabular}{lll}
\hline & Diskurskohärenz & Satzkohärenz \\
\hline Faktizitätskontexte & Tempora & temporale Konnektoren \\
\hline $\begin{array}{l}\text { Wahrheits- und } \\
\text { Erwünschtheitskontexte }\end{array}$ & Modi des Verbs & $\begin{array}{l}\text { epistemische und deontische } \\
\text { Konnektoren }\end{array}$ \\
\hline
\end{tabular}

Vor dem Hintergrund dieser Überlegungen kann die in Blühdorn/Reichmann (2010, 35s.) angedeutete Hypothese zur funktionalen Differenz zwischen finiten adverbialen Nebensätzen und adverbialen Infinitivgruppen präzisiert werden. 
Adverbiale Infinitivgruppen sind funktional auf Beiträge zur Satzkohärenz festgelegt. Sie können keine selbständigen Propositionen und/oder Sprechakte bilden, können keine Beiträge zur Diskurskohärenz leisten, können insbesondere selbst nicht in epistemischer Relation zum Sprecherwissen und in deontischer Relation zum Normen- und Intentionensystem des Sprechers ausgewertet werden. Sie sind jedoch nicht darauf beschränkt, für ihren Matrixsatz Faktizitätskontexte bereitzustellen und damit Sachverhaltsverknüpfungen zu stiften. Sie können ihren Matrixsatz durchaus auch epistemisch und/oder deontisch kontextualisieren, indem sie z.B. Bedingungen, Motive oder Ziele für den ausgeführten Sprechakt benennen.

Finite adverbiale Nebensätze leisten demgegenüber stets sowohl zur Satzkohärenz als auch zur Diskurskohärenz Beiträge. Sie können selbständige Propositionen und/oder Sprechakte bilden und können sowohl auf der Satzebene als auch auf der Diskursebene Faktizitäts-, Wahrheits- und/oder Erwünschtheitskontexte bereitstellen.

Im Deutschen, wo finite Nebensätze viel häufiger gebraucht werden als satzwertige Infinitivgruppen, leisten verbhaltige Adverbialia somit im Regelfall nicht nur Beiträge zur Satzkohärenz, sondern auch Beiträge zur Diskurskohärenz. Verknüpfungen mit verbalen Ausdrücken ohne Rückbindung an den Sprecher können vor allem dann gewählt werden, wenn der beschriebene Sachverhalt im relevanten Kontext nicht faktisch ist, wie es die Präpositionen ohne und (an)statt sowie finales um anzeigen. Für reine Satzverknüpfungen ohne Rückbindung an den Sprecher werden im Deutschen bevorzugt Präpositionalphrasen mit deverbalen Nominalisierungen verwendet (nach dem Ende der letzten Vorlesung, zu Ihrer Kenntnisnahme, aufgrund der oben dargestellten Zusammenhänge etc.).

Im Portugiesischen können für Verknüpfungsrelationen aller Art verbhaltige Adverbialia mit oder ohne Diskursanbindung gewählt werden. Finite adverbiale Nebensätze haben in portugiesischen Texten typischerweise einen etwas geringeren Anteil als in deutschen. Für reine Satzverknüpfungen ohne Diskursanbindung werden verbhaltige Ausdrücke (Infinitiv-, Gerundial- und Partizipialgruppen) gegenüber präpositionalen Adverbialia mit deverbalen Nominalisierungen eindeutig bevorzugt (cf. Blühdorn 2012b). Ein typisches Beispiel:

(27) Os familiares da atriz pediram que os amigos dela, em vez de enviar flores, façam doações à Casa do Teatro. (operamundi.uol.com.br)

(27a) Die Familie der Schauspielerin bat anstelle von Beileidsbekundungen um Spenden für das Schauspielhaus. 
Aufgrund von Beobachtungen an längeren Passagen fiktionaler und nicht-fiktionaler Texte beider Sprachen, aus denen die in diesem Aufsatz verwendeten Belege stammen, lässt sich - ohne hier Details zu dokumentieren - abschätzen, dass die quantitative Verteilung der Ausdrucksklassen für Adverbialverknüpfungen im Portugiesischen und Deutschen ungefähr der folgenden Abbildung entspricht:

Abb. 3: Unterschiedliche Nutzung von Ausdrucksklassen für Adverbialverknüpfungen (geschätzt)

\begin{tabular}{l|l}
\hline Portugiesisch & Deutsch \\
\hline \multirow{2}{*}{ finite Nebensätze } & \multirow{2}{*}{ finite Nebensätze } \\
\hline \multirow{2}{*}{ infinite verbale Ausdrücke } & \\
& infinite verbale Ausdrücke \\
deverbale Nominalisierungen & deverbale Nominalisierungen \\
\hline
\end{tabular}

\section{6 Übersetzung}

Im Allgemeinen wird davon ausgegangen, dass die Aufgabe des Übersetzers nicht in erster Linie darin besteht, grammatische Strukturen der Ausgangssprache durch grammatische Entsprechungen der Zielsprache wiederzugeben, sondern vor allem darin, einen zielsprachlichen Text zu entwickeln, der dem Ausgangstext in funktionaler Hinsicht möglichst nahekommt (cf. Nord 2010, Snell-Hornby 2006, 51-55). Anhand der kontrastiven Analyse kleinerer Übersetzungseinheiten lässt sich nachvollziehen, ob die Funktion bestimmter grammatischer Strukturen erkannt und in der Übersetzung (ggf. mit anderen sprachlichen Mitteln) adäquat wiedergegeben wurde (cf. Reichmann 2005). Daher kann es auch in diesem Zusammenhang erhellend sein, sich anhand einiger Beispiele vor Augen zu führen, wie adverbiale Nebensätze und Infinitivgruppen vom Deutschen ins Portugiesische und umgekehrt übersetzt werden. Als Datengrundlage dienen die Romane Dois irmãos des brasilianischen Schriftstellers Milton Hatoum und Der Vorleser von Bernhard Schlink sowie ihre Übersetzungen in die jeweils andere Sprache.

Die Texte zeigen, dass die Übersetzer im Grundsatz dazu tendieren, analoge grammatische Strukturen zu nutzen, wenn die Sprachen über sie verfügen. So werden z.B. adverbiale Infinitivgruppen mit para typischerweise durch um-Infini- 
tive und finite adverbiale Nebensätze mit para que durch damit-Sätze wiedergegeben:

(28) Yaqub se aproximou, mirou de perto a fotografia para enxergar as feições do irmão (...). (Dois Irmãos, 17)

(29) Yaqub trat vor, betrachtete die Fotografie aus der Nähe, um die Gesichtszüge des Bruders (...) genauer zu erkennen (...). (Zwei Brüder, 19)

(30) Mas (...) [eu] soube que [ela] ergueu a cabeça e perguntou em árabe para que só a filha e a amiga quase centenária entendessem (...). (Dois Irmãos, 10)

(31) Doch erfuhr ich, dass sie (...) den Kopf anhob und auf arabisch, damit nur ihre Tochter und die fast hundertjährige Freundin es verstanden, fragte (...). (Zwei Brüder, 10)

Dagegen werden Infinitivgruppen mit antes oder por, die keine analoge Entsprechung im Deutschen finden, oft durch adverbiale Nebensätze wiedergegeben:

(32) Antes de abandonar a casa, Zana via o vulto do pai e do esposo nos pesadelos das últimas noites (...). (Dois Irmãos, 9)

(33) Bevor sie das Haus endgültig verließ, sah sie in den Alpträumen der letzten Nächte die Gestalten ihres Vaters und ihres Mannes (...). (Zwei Brüder, 9)

(34) E lá estava Zana, impávida na rede vermelha, (...) entristecida por passar mais uma noite sem o filho. (Dois Irmãos, 26)

(35) Und da lag Zana ungerührt in der roten Hängematte, (...) zerquält, weil sie wieder eine Nacht ohne ihren Sohn verbracht hatte. (Zwei Brüder, 30)

In solchen Beispielen führt die Hinzufügung von Tempus und Modus im Deutschen zu einer Diskursanbindung, die im Ausgangstext fehlt. In (32) und (34) zeigen die Infinitivgruppen lediglich eine Relation von Ursache und Wirkung zwischen zwei Sachverhalten an (Satzkohärenz). In den Übersetzungen wird durch die finiten Verbformen eine Rückbindung an den Sprecher und damit an den Diskurs hinzugefügt. In (35) wird durch die Wahl der finiten Form verbracht hatte der Sinn des Kausalsatzes gegenüber dem Ausgangstext auf eine von mehreren Lesarten reduziert. Der Ausgangstext ermöglicht neben der in der Übersetzung gegebenen Interpretation auch Deutungen, die durch Formulierungen wie weil sie wieder eine Nacht ohne ihren Sohn verbrachte oder weil sie wieder eine Nacht ohne ihren Sohn verbringen musste wiederzugeben wären. Der Infinitiv Präsens im portugiesischen Text legt sicherlich die erste dieser Deutungen besonders nahe, schließt aber auch die zweite und letztlich auch diejenige, die die 
Übersetzerin gewählt hat, nicht aus. Diese Vieldeutigkeit ist eine Folge der fehlenden Rückbindung an den Diskurs. Bei der Übertragung ins Deutsche ist der Übersetzer gezwungen, eine der möglichen Lesarten auszuwählen und die übrigen wegfallen zu lassen, weil der Infinitiv als neutrale Ausdrucksvariante hier nicht zur Verfügung steht.

In der umgekehrten Übersetzungsrichtung werden adverbiale Nebensätze gelegentlich durch Infinitivgruppen wiedergegeben. Dann wird die im Ausgangstext vorhandene Rückbindung an den Sprecher eliminiert. In der Übersetzung wird nurmehr Satzkohärenz hergestellt:

(36) Ich (...) meinte, sie zu spüren, bis ich merkte, dass ich das Kissen oder die Decke hielt. (Der Vorleser, 28)

(37) (...) [eu] pensava senti-la até reparar que estava segurando o travesseiro ou o cobertor. (O leitor, 33)

In vielen Fällen ergibt sich durch die Wahl einer Infinitivgruppe anstelle eines finiten Nebensatzes faktisch keine größere Interpretationsbreite. Die formale Rückbindung an den Diskurs durch Tempus und Modus wird auch im Deutschen keineswegs immer für die Interpretation genutzt. In Beispielen wie (36) bietet das Deutsche schlicht keine Ausdrucksalternative zum finiten Nebensatz. Die Diskursrückbindung kann hier faktisch auch als redundant betrachtet werden und uninterpretiert bleiben.

Im Portugiesischen wäre als Variante zur Infinitivgruppe in (37) ein finiter Nebensatz möglich: até que reparasse que estava segurando o travesseiro. Solche Formulierungen wirken aber leicht überexplizit und stilistisch schwerfällig, wenn für die Diskursrückbindung kein eindeutiger Bedarf erkennbar ist.

\section{Zusammenfassung und Ausblick}

In diesem Beitrag wurde der Unterschied zwischen finiten adverbialen Nebensätzen und adverbialen Infinitivgruppen im Hinblick auf ihre Funktionen für Informationsstruktur und Textkohärenz untersucht.

Wenn man von deverbalen Nominalisierungen absieht, stehen im Deutschen zwei Ausdrucksoptionen zur Verfügung. Typisch sind finite Nebensätze, die ein eigenes hervorhebbares Subjekt enthalten und durch Tempus- und Modusmarkierung stets eine eigene Diskursverankerung bewirken. Die deutlich seltenere Alternative sind Infinitivgruppen, denen beide Eigenschaften fehlen.

Im Portugiesischen bestehen drei Ausdrucksoptionen. Finite Nebensätze sind funktional ebenso zu kennzeichnen wie für das Deutsche, werden aber anteilig 
seltener verwendet. Sehr viel häufiger werden Infinitivgruppen ${ }^{3}$ gebraucht, bei denen keine eigene Diskursverankerung stattfindet. Da Infinitivgruppen im Portugiesischen eigene Subjekte haben können, geht damit nicht automatisch eine Verminderung der Ausdrucksmöglichkeiten im Bereich der Informationsstruktur einher.

Das Verhältnis zwischen finiten adverbialen Nebensätzen und adverbialen Infinitivgruppen, das manchen Grammatikern Kopfzerbrechen bereitet hat, konnte mit dieser Untersuchung ein Stück weit aufgehellt werden. Es wurde deutlich, dass es sich nicht um reine Ausdrucksalternativen ohne Funktionsunterschied handelt, sondern dass eine Sprache wie das Portugiesische, die beide Optionen vielfältig nutzt, im Bereich der verbhaltigen Adverbialia funktionale Unterscheidungen ermöglicht, die im Deutschen so nicht gemacht werden können. In der Praxis der Übersetzung kann das dazu führen, dass vom Portugiesischen ins Deutsche Diskursanbindungen hinzugefügt werden, die die Interpretationsbreite verengen, während vom Deutschen ins Portugiesische Diskursanbindungen getilgt werden, wodurch die Übersetzung ökonomischer erscheint als der Ausgangstext.

\section{Bibliographie}

Becker, Martin G./Remberger, Eva-Maria (edd.), Modality and Mood in Romance. Modal interpretation, mood selection, and mood alternation, Berlin, De Gruyter, 2010.

Bierwisch, Manfred, Heads, complements, adjuncts. Projection and saturation, in: Lang, Ewald/ Maienborn, Claudia/Fabricius-Hansen, Cathrine (edd.), Modifying adjuncts, Berlin, Mouton de Gruyter, 2003, 113-159.

Blühdorn, Hardarik, Syntax und Semantik der Konnektoren. Ein Überblick, Mannheim, Institut für Deutsche Sprache, 2008, online unter: <http://www.ids-mannheim.de/gra/texte/blu_ueberblick.pdf>.

Blühdorn, Hardarik, Negation im Deutschen. Syntax, Informationsstruktur, Semantik, Tübingen, Narr, 2012 (= 2012a).

Blühdorn, Hardarik, Zur Syntax adverbialer Satzverknüpfungen. Deutsch - Italienisch - Portugiesisch, in: Gunkel, Lutz/Zifonun, Gisela (edd.), Deutsch im Sprachvergleich. Grammatische Kontraste und Konvergenzen, Berlin, De Gruyter, 2012, 301-331 (= 2012b).

Blühdorn, Hardarik/Reichmann, Tinka, Modal readings of sentence connectives in German and Portuguese, in: Becker/Remberger (2010), 15-38.

3 Kortmann $(1997,243)$ beobachtet im Hinblick auf die Verwendung verbhaltiger Adverbialia arealtypologische Unterschiede zwischen Zentrum und Peripherie des mitteleuropäischen Sprachraums: Im Zentrum werden finite Nebensätze bevorzugt, an der Peripherie nimmt die Nutzung infiniter verbhaltiger Ausdrücke zu. 
Corver, Norbert/Delfitto, Denis, Introduction: on adverbs and adverbial modification, Italian Journal of Linguistics/Rivista di Linguistica 12 (2000), 3-11.

Cunha, Celso/Cintra, Lindley, Nova Gramática do Português Contemporâneo, Rio de Janeiro, Lexikon, ${ }^{5} 2008$.

Delfitto, Denis, Adverbs and the Syntax/Semantics Interface, Italian Journal of Linguistics/Rivista di Linguistica 12 (2000), 13-53.

Diewald, Gabriele, Die Modalverben im Deutschen. Grammatikalisierung und Polyfunktionalität, Tübingen, Niemeyer, 1999.

Duden, Die Grammatik. Unentbehrlich für richtiges Deutsch, Mannheim, Dudenverlag, ${ }^{8} 2009$.

Frawley, William, Linguistic Semantics, Hillsdale, N.J., Erlbaum, 1992.

Gärtner, Eberhard, Konjunktion = Konjunktion, Präposition = Präposition $?$ Eine vergleichende Untersuchung zu Funktionswörtern im Deutschen und Portugiesischen, in: Blühdorn, Hardarik/Schmidt-Radefeldt, Jürgen (edd.), Die kleineren Wortarten im Sprachvergleich DeutschPortugiesisch, Frankfurt/Main, Lang, 2003, 89-113.

Klein, Wolfgang, Time in Language, London, Routledge, 1994.

Kortmann, Bernd, Adverbial Subordination. A typology and history of adverbial subordinators based on European languages, Berlin, Mouton de Gruyter, 1997.

Lohnstein, Horst, Variable und invariante Strukturmerkmale von Satzkonnektoren, in: Blühdorn, Hardarik/Breindl, Eva/Waßner, Ulrich Hermann (edd.), Brücken schlagen. Grundlagen der Konnektorensemantik, Berlin, De Gruyter, 2004, 137-160.

Lyons, John, Semantics, Cambridge, Cambridge University Press, 1977.

Marques, Rui, Modality, context change potential and mood selection in European Portuguese, in: Becker/Remberger (2010), 133-161.

Neves, Maria Helena de Moura, Gramática de Usos do Português, São Paulo, Editora UNESP, 2000.

Nord, Christiane, Funktionsgerechtigkeit und Loyalität. Theorie, Methode und Didaktik des funktionalen Übersetzens, vol. 1, Berlin, Frank \& Timme, 2010.

Peres, João Andrade/Mascarenhas, Salvador, Notes on sentential connections (predominantly) in Portuguese, Journal of Portuguese Linguistics 5 (2006), 113-169.

Reichmann, Tinka, Satzspaltung und Informationsstruktur im Portugiesischen und im Deutschen. Ein Beitrag zur kontrastiven Linguistik und Übersetzungswissenschaft, Frankfurt am Main, Lang, 2005.

Rocha, Maura A. Freitas/Lopes, Ruth E. Vasconcellos, Adjunção, in: Gramática do Português Culto Falado no Brasil (coord. geral: Ataliba T. de Castilho), vol. III: Kato, Mary A./do Nascimento, Milton (edd.), A Construção da Sentença, Campinas, SP, Editora da UNICAMP, 2009, 193-236.

Rudolph, Elisabeth, Gemeinsamkeiten und Unterschiede im literarischen Gebrauch von Satzverknüpfungen im Spanischen, Portugiesischen und Deutschen, in: Lüdtke, Helmut/SchmidtRadefeldt, Jürgen (edd.), Linguistica contrastiva. Deutsch versus Portugiesisch - Spanisch Französisch, Tübingen, Narr, 1997, 325-348.

Snell-Hornby, Mary, The Turns of Translation Studies. New paradigms or shifting viewpoints?, Amsterdam, Benjamins, 2006.

Squartini, Mario, Where mood, modality and illocution meet: the morphosyntax of Romance conjectures, in: Becker/Remberger (2010), 109-130.

Sweetser, Eve, From etymology to pragmatics. Metaphorical and cultural aspects of semantic structure, Cambridge, University Press, 1990. 
Vilela, Mário/Koch, Ingedore Villaça, Gramática da Língua Portuguesa. Gramática da PalavraGramática da Frase - Gramática do Texto/Discurso, Coimbra, Almedina, 2001.

\section{Quellen für Abschnitt 6}

Hatoum, Milton, Dois Irmãos, São Paulo, Companhia das Letras, 2000.

Hatoum, Milton, Zwei Brüder (übersetzt von Karin von Schweder-Schreiner), Frankfurt am Main, Suhrkamp, 2002.

Schlink, Bernhard, Der Vorleser, Zürich, Diogenes, 1995.

Schlink, Bernhard, $O$ leitor (tradução de Pedro Süssekind), Rio de Janeiro, Record, 2010. 